\title{
Deactivation vs. asynchronous pacing - prospective evaluation of a protocol for rhythm management in patients with magnetic resonance conditional pacemakers undergoing adenosine stress cardiovascular magnetic resonance imaging
}

\author{
Oliver Klein-Wiele ${ }^{1 *}$ (D), Marietta Garmer ${ }^{2}$, Gianluca Barbone', Rhyan Urbien', Martin Busch², Kaffer Kara ${ }^{3}$, \\ Harald Schäfer ${ }^{1}$, Michael Schulte-Hermes ${ }^{1,4}$, Birgit Hailer ${ }^{1}$ and Dietrich Grönemeyer ${ }^{2}$
}

\begin{abstract}
Background: Cardiovascular Magnetic Resonance (CMR) imaging with adenosine stress is an important diagnostic tool in patients with known or suspected coronary artery disease (CAD). However, the method is not yet established for CAD patients with pacemakers (PM) in clinical practice. A possible reason is that no recommendations exist for PM setting (paused pacing or asynchronous mode) during adenosine stress. We elaborated a protocol for rhythm management in clinical routine for PM patients that considers heart rate changes under adenosine using a test infusion of adenosine in selected patients.

Methods: 47 consecutive patients (mean age $72.3 \pm$ 10,0 years) with MR conditional PM and known or suspected CAD who underwent CMR in clinical routine were studied in this prospective observational study. PM indications were sinus node dysfunction (SND, $n=19 ; 40,4 \%$ ), atrioventricular (AV) block ( $n=26 ; 55.3 \%)$ and bradyarrhythmia in permanent atrial fibrillation (AF, $n=2 ; 4.3 \%$ ). In patients with SND, normal AV-conduction and resting HR $>45 \mathrm{bpm}$ at the time of CMR and in AF the PM was deactivated for the scan. In intermittent AV-block a test infusion of adenosine was given prior to the scan. All patients with permanent higher degree sinuatrial or AV-block or deterioration of AV-conduction in the adenosine test were paced asynchronously during CMR, in patients with preserved AV-conduction under adenosine the pacemaker was deactivated. CMR protocol included cine imaging, adenosine stress perfusion and late gadolinium enhancement.

Results: The adenosine test was able to differentiate between mandatory PM stimulation during CMR and safe deactivation of the device. In patients with permanent sinuatrial or AV-block $(n=11 ; 23.4 \%)$ or deterioration of AV conduction in the adenosine test $(n=5,10.6 \%)$ asynchronous pacing above resting heart rate did not interfere with intrinsic rhythm, no competitive stimulation was seen during the scan. 10 of 15 (66,7\%) patients with intermittent AV-block showed preserved AV-conduction under adenosine. As in SND and AF deactivation of the PM showed to be safe during CMR, no bradycardia was observed.

(Continued on next page)
\end{abstract}

\footnotetext{
* Correspondence: o.klein-wiele@kk-essen.de

'Department of Cardiology, Katholisches Klinikum Essen, University of

Witten/Herdecke, Hülsmannstraße 17, 45355 Essen, Germany

Full list of author information is available at the end of the article
} 
(Continued from previous page)

Conclusion: Our protocol for rhythm management during adenosine stress CMR showed to be feasible and safe and may be recommended for pacemaker patients undergoing routine CMR.

Keywords: Pacemaker, MRI conditional, Cardiovascular magnetic resonance, CMR, Adenosine stress, Safety, Sinus node dysfunction, Atrioventricular block, Asynchronous pacing

\section{Background}

CMR as a non-invasive imaging modality is firmly established in the clinical workup for patients with known or suspected CAD. Beside cine imaging and scar detection with Late Gadolinium Enhancement (LGE) ischemia detection plays a major role in CAD $[1,2]$. It has a class Ia level A recommendation in case of intermediate pre test probability of CAD in latest guidelines [2]. Hemodynamic relevance of stenoses in known CAD can be evaluated reliably [3].

In patients with cardiac conduction disorders underlying or concomitant CAD is common $[4,5]$. However, while safety of CMR without stress agents in patients with MR conditional PM has been shown in a number of studies [6-8] no prospective data exists on rhythm management of those patients in adenosine stress CMR.

In MR conditional PM no inhibited mode can be chosen. Only deactivation (ODO-mode) or asynchronous pacing (DOO, AOO, VOO) [9] are available to avoid inhibition by electromagnetic interference or tracking of electromagnetic impulses. In SND and AV-block selecting an adequate pacing mode for routine adenosine stress CMR can be challenging because the effect of adenosine on heart rate (HR) has not been thoroughly studied in this entity of patients. Asynchronous mode (i.e. pacing at a fixed rate above baseline HR) could result in competitive pacing when HR accelerates under adenosine [10] reaching the pacing rate. This is due to the fact that sensing is deactivated in this mode and intrinsic rhythm cannot inhibit PM activity. PM stimuli falling in the vulnerable period of the cardiac cycle could then trigger arrhythmia [11]. On the other hand deactivation of the PM in patients with normal HR under resting conditions could result in bradycardia or asystole under adenosine administration [12].

We recently published first data on adenosine stress CMR in pacemaker patients in a small retrospective study and found no relevant complications under a predefined pacing protocol [13]. However, the number of patients with AV-block was limited and it remains unclear what pacing mode is preferable for patients with intermittent AV-block i.e. without higher degree AVblock at the time of CMR. To further investigate adenosine response and to decide whether the pacemaker can be deactivated during the scan we used a test infusion of adenosine for patients with intermittent AV-block and preserved AV-conduction at the time of CMR to screen for adenosine induced bradycardia.

In conclusion the diagnostic value of perfusion analysis by CMR in CAD is well known but still no recommendations exist for PM programming in MRI conditional devices during adenosine stress. We sought to test the safety of a protocol for rhythm management in those patients that is adapted to the adenosine response in PM patients with different underlying conduction disorders.

\section{Methods}

In this prospective observational study we analyzed 47 consecutive patients with MR conditional PM who underwent routine adenosine stress CMR including cine imaging, adenosine stress perfusion and LGE from april 2015 to december 2016. Inclusion criteria were presence of a MR conditional pacemaker system, indication for adenosine stress CMR for the evaluation of known or suspected CAD, age $>18$ years. Exclusion criteria were implantation $<6$ weeks from the scan, epicardial or abandoned pacemaker leads, bronchial, asthma, impaired renal function, ejection fraction $<35 \%$, presence of an antitachycardia device.

\section{General characteristics}

Patients had a mean age of $72.1 \pm 11.0$ years. 19 (40.4\%) had known CAD, 8 (17.0\%) previous MI. All other patients had intermediate pretest probability of CAD [14]. Echocardiography had shown preserved systolic left ventricular (LV) function in all subjects. Pacemaker indications were sinus node dysfunction (SND, 19; 40.4\%), second or third degree AV-block $(n=26 ; 55.3 \%)$ and bradyarrhythmia in permanent $\operatorname{AF}(n=2 ; 4.3 \%)$. Eight patients (17.0\%) were PM dependent (HR $<30 \mathrm{bpm})$. Impulse generator/lead models were Advisa $(n=1 ; 2.1 \%)$ and Ensura $(n=46 ; 97.9 \%)$ MRI SureScan/CapSureFix 5076 Novus (atrial), CapSureSense 4074 (ventricular) (Medtronic Inc., Minneapolis, MA, USA). For detailed baseline characteristics see Table 1 .

\section{Pacemaker programming and adenosine test}

CMR was performed more than six weeks after PM implantation in all individuals according to ESC guidelines [15]. Prior to and after CMR imaging battery status of the device, lead impedance, pacing capture thresholds and sensing amplitudes were measured. In patients with 
Table 1 Baseline characteristics

\begin{tabular}{lll}
\hline Total patients & 47 & \\
\hline Mean Age (years) & $72.3 \pm 10.0$ & $\%$ \\
Female & N & 46.8 \\
Pacemaker indication & & \\
Higher degree AV Block & 26 & 55.3 \\
Sinus node dysfunction & 19 & 40.4 \\
Bradyarrhythmia in AF & 2 & 4.3 \\
Coronary artery disease & 19 & 40.4 \\
Previous MI & 8 & 17.0 \\
Paroxysmal atrial fibrillation & 17 & 36.2 \\
In AF at the time of CMR & 2 & 4.3 \\
Hypertension & 40 & 85.1 \\
Impaired renal function & 2 & 4.3 \\
Previous Stroke & 7 & 15.0 \\
Pacemaker & & \\
Ensura MRI Sure Scan & 46 & 2.1 \\
Advisa DR MRI Sure Scan & 1 & 17.0 \\
Pacemaker dependent & 8 &
\end{tabular}

$\mathrm{AV}$, atrioventricular, $\mathrm{AF}$, atrial fibrillation, $\mathrm{Ml}$, myocardial infarction, $\mathrm{CMR}$, cardiovascular magnetic resonance

intermittent $\mathrm{AV}$-block and preserved $\mathrm{AV}$-conduction at the time of CMR PQ-interval was measured in resting ECG and the Wenckebach point was assessed by testing in $\mathrm{AOO}$ mode via the PM programmer. A Wenckebach point below $120 \mathrm{bpm}$ was considered pathologic. Furthermore these patients received a test infusion of adenosine as 3-min infusion of $140 \mu \mathrm{g} / \mathrm{kg}$ body weight $/ \mathrm{min}$ according to the dose for CMR. The pacemaker was deactivated for the test (ODO). Possible deterioration of $\mathrm{AV}$-conduction was recorded and classified in progression to second degree AV-block II Mobitz I, second degree AV-block Mobitz II and third degree AV-block. Reactivation of pacing was performed immediately in case of bradycardia. In SND the PQ interval and the Wenckebach point were measured to rule out occult AV-nodal disease; as in AF no adenosine test was performed prior to MR.

Devices were programmed to MR safe mode following manufacturer's instructions immediately prior to the scan and reprogrammed immediately thereafter. Programming was performed according to a predefined protocol: To avoid interference of intrinsic rhythm with PM-stimulation in patients with SND and resting heart rate $\mathrm{HR}>45 \mathrm{bpm}$ no pacing (ODO)-mode was engaged during the scan - also when atrial fibrillation (AF) was present. In individuals with SND and HR $\leq 45 \mathrm{bpm}$ the pacemaker was set to asynchronous atrial stimulation (AOO, $60 \mathrm{bpm}$ ). All patients with permanent second or third degree AV block and those with deterioration of $\mathrm{AV}$-conduction in the adenosine test were continuously paced in asynchronous mode. Pacing rate was set $10 \mathrm{bpm}$ above spontaneous heart rate with a minimum of $60 \mathrm{bpm}$. VOO mode at $60 \mathrm{bpm}$ was chosen in AV block with sinus rate $>45 \mathrm{bpm}$ to avoid competitive atrial stimulation, DOO mode at $60 \mathrm{bpm}$ in AV block with sinus bradycardia $\leq 45 \mathrm{bpm}$. In patients with intermittent AV-block and preserved AV-conduction (i.e. no progression to second or third degree AV-block in the adenosine test) the PM was deactivated as in SND. Patients in $\mathrm{AF}$ at the time of the scan were paced $\mathrm{VOO}$ at $60 \mathrm{bpm}$ if resting heart rate was $\leq 45 \mathrm{bpm}$. Table 2 shows the protocol used to select pacing modes for specific clinical constellations.

\section{Safety precautions}

As previously described [13] patients were monitored during the scan with continuous electrocardiographic and visual supervision by a cardiologist present in the scanner room. Voice contact was maintained with the patient at all times of the scan. Advanced cardiac life support protocol was in effect. In the scanner the patient was placed on a carry sheet; medical staff was trained for rapid removal of the patient from the scanner room in the event of cardiopulmonary compromise. Thus immediate treatment of severe arrhythmia and reactivation of PM stimulation within seconds in non-paced patients was guaranteed. Atropine, adrenaline and theophylline injections were prepared ready for use in case of bradycardia. Two separate cubital venous cannulas were used for adenosine and gadolinium contrast agent respectively.

\section{Cardiac magnetic resonance imaging}

CMR scans were performed with a $1.5 \mathrm{~T}$ wide bore system (ESPREE - Siemens Healthcare, Erlangen, Germany) using a 4-channel body array and an 8-channel spine coil. Maximum gradient field was $33 \mathrm{mT} / \mathrm{m}$ (Z-Engine) with a slew rate of $100 \mathrm{~T} / \mathrm{m} / S$. maximum specific absorption rates were limited to $2.0 \mathrm{~W} / \mathrm{kg}$.

As previously published [13] our standard protocol meets the Society of Cardiovascular Magnetic Resonance (SCMR) guidelines for CMR [16]. Cine steady-state free precession (SSFP) gradient-echo images were obtained in 10 to 12 short axis slices depending on the size of the ventricles and in 3 long axis planes corresponding to two, three and four chamber views. For stress perfusionimaging adenosine was administered as 3-min infusion of $140 \mu \mathrm{g} / \mathrm{kg}$ body weight $/ \mathrm{min}$. First-pass perfusion imaging was carried out with intravenous bolus administration of gadolinium $(0.2 \mathrm{mmol} / \mathrm{kg}$ body weight $)$ in a fast low angle shot (FLASH) sequence (3 to 4 slices). Late Gadolinium Enhancement (LGE) images were acquired fifteen minutes after injection of gadolinium as phase- 
Table 2 Protocol for the selection of pacing modes

\begin{tabular}{ll}
$\begin{array}{l}\text { Sinus node dysfunction } \\
\text { without AV-block }\end{array}$ & ODO; AOO at $60 \mathrm{bpm}$ when IHR $<45 \mathrm{bpm}$ \\
\hline $\begin{array}{l}\text { Atrial fibrillation } \\
\text { at time of scan }\end{array}$ & VOO at $60 \mathrm{bpm}$ when $\mathrm{HR}<45 \mathrm{bpm}$ \\
\hline $\begin{array}{l}\text { AV-block }>1^{\circ} \\
\text { (present) }\end{array}$ & $\begin{array}{l}\text { VOO at } 10 \mathrm{bpm}>\mathrm{IHR} \text { when } \mathrm{SR}>45 \mathrm{bpm}, \text { minimum } 60 \mathrm{bpm} \\
\text { DOO at } 10 \mathrm{bpm}>\mathrm{IHR} \text { when } \mathrm{SR}<45 \mathrm{bpm}, \text { minimum } 60 \mathrm{bpm}\end{array}$
\end{tabular}

Intermittent AV-block, preserved AV-conduction present

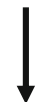

Adenosine test ( $3 \mathrm{~min}$. infusion at $140 \mathrm{ug} / \mathrm{kg}$ body weight $/ \mathrm{min}$ )

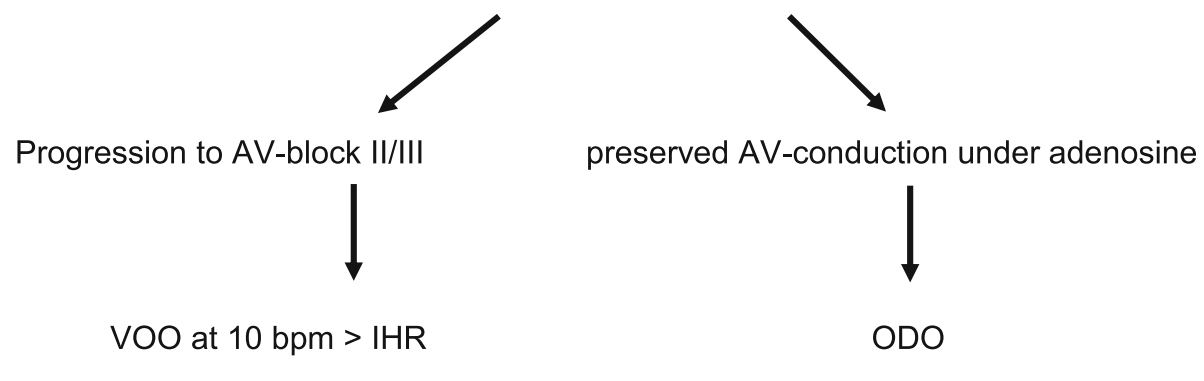

$\mathrm{AV}$, atrioventricular; bpm, beats per minute; IHR, intrinsic heart rate; SR, sinus rhythm

sensitive inversion-recovery (PSIR) in short (10 to 12 slices) and long axis (3 planes) views.

\section{Results}

Adenosine test prior to CMR

15 patients (31.9\%) with intermittent AV-block and preserved $\mathrm{AV}$-conduction at the time of CMR were tested. 5 of them (33.3\%) showed deterioration of AV conduction with a drop of HR. All of those individuals had both prolonged PQ-interval (220 to $305 \mathrm{~ms}$ ) and pathologic Wenckebach point (70 to $90 \mathrm{bpm}$ ). Two patients showed worsening of AV-conduction to AV-block II Mobitz II, three patients developed AV-block III. One patient had pathologic PQ-interval (230 ms) and normal Wenckebach point $(130 \mathrm{bpm})$ and showed acceleration of HR from 70 to $85 \mathrm{bpm}$ without progressive AV-block. One patient had normal AV-conduction $(190 \mathrm{~ms})$ and pathologic Wenckebach point $(115 \mathrm{~ms})$ and showed an increase in HR from 85 to $95 \mathrm{bpm}$. Seven of 15 patients with intermittent AV-block (46.6\%\%) had normal PQinterval and normal Wenckebach point. None of them showed higher degree AV-block in the test, heart rate increased from $66 \pm 8.6$ to $75 \pm 11.4 \mathrm{bpm}(p<0.001$, paired t-test). Thus only in patients with both pathologic
PQ-interval and pathologic Wenckebach point worsening of AV-conduction and drop of HR were observed. The adenosine test was well tolerated by the patients; no adverse reactions were seen. Figure 1 summarizes heart rate response under the test infusion of adenosine.

\section{Effect and safety of adenosine administration for stress perfusion}

There were no adenosine induced adverse events. Asynchronous stimulation of patients with permanent AV-block or progressive AV-block under adenosine $10 \mathrm{bpm}$ above resting $\mathrm{HR}$ did not cause competitive pacing or arrhythmia during MR. Patients with preserved AV-conduction under adenosine and deactivated PM did not develop higher degree AV-block in CMR, $\mathrm{HR}$ increased from $67.6 \pm 9.1$ to $77.6 \pm 11.3 \mathrm{bpm}$. Two patients with SND were in AF at the time of CMR. In patients with SND and normal AV-conduction i.e. normal PQ interval and normal Wenckebach point $(n=17$; $36.2 \%)$ at the time of the scan with deactivated (ODO) pacemaker adenosine administration accelerated HR from $60.1 \pm 9.1$ to $76.0 \pm 9.3 \mathrm{bpm}(p<0.001$, Wilcoxon signed rank test). AV-conduction was not significantly influenced by adenosine; no higher degree AV block 


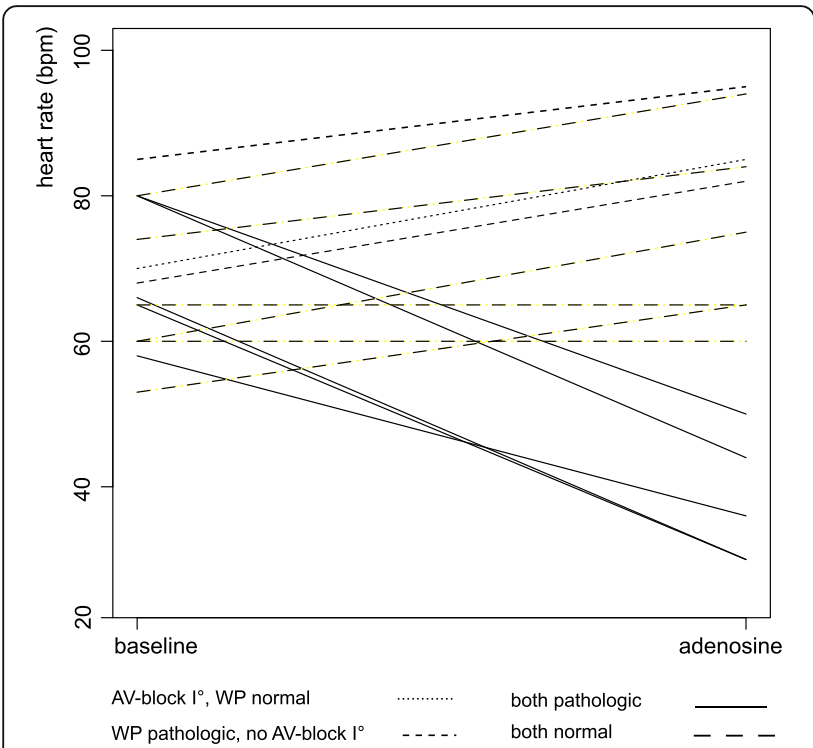

Fig. 1 Adenosine test in intermittent AV-block. Individual changes of heart rate in patients with intermittent AV-block and currently preserved AV-conduction under a 3 min infusion of adenosine at $140 \mu \mathrm{g} / \mathrm{kg}$ body weight/min. PQ-interval and Wenckebach point was assessed before the test and is indicated by different lines. Progression to AV-block II/III was only observed in patients with both prolonged PQ-interval (AV-block $I^{\circ}$ ) and pathologic Wenckebach point (< 120 bpm). AV, atrioventricular; WP, Wenckebach point; bpm, beats per minute

occurred. When sinus rate was $<45 \mathrm{bpm}(n=1 ; 2.1 \%)$ $\mathrm{AOO}$ pacing at $60 \mathrm{bpm}$ led to permanent capture, no acceleration of $\mathrm{HR}$ under adenosine was noticed. Of 4 patients in $\mathrm{AF}$ in $3 \mathrm{HR}$ remained unchanged during stress perfusion, one patient had acceleration of HR from 110 to $125 \mathrm{bpm}$.

\section{Device integrity}

Device integrity was not compromised by the CMR scan. No patient showed relevant alterations of lead impedance, pacing capture threshold, sensing amplitude or battery voltage. Table 3 summarizes device parameters pre and post CMR.

Table 3 Comparison of device parameters before and after CMR

\begin{tabular}{llll}
\hline & before MR & after MR & $P^{\mathrm{a}}$ \\
\hline P-wave amplitude (mV) & $2.87 \pm 1.86$ & $3.10 \pm 1.70$ & 0.32 \\
R-wave amplitude (mV) & $12.27 \pm 5.32$ & $12.05 \pm 5.44$ & 0.59 \\
Atrial lead impedance (Ohm) & $469 \pm 61$ & $468 \pm 65$ & 0.65 \\
Ventricular lead impedance (Ohm) & $601 \pm 120$ & $603 \pm 118$ & 0.57 \\
Atrial PCT (V@0.4 ms) & $0.66 \pm 0.25$ & $0.66 \pm 0.20$ & 1.0 \\
Ventricular PCT (V@0.4 ms) & $0.63 \pm 0.26$ & $0.55 \pm 0.28$ & 0.1 \\
Battery voltage (V) & $2.97 \pm 0.42$ & $2.97 \pm 0.42$ & n.a. \\
\hline
\end{tabular}

CMR, Cardiovascular Magnetic Resonance; PCT, pacing capture threshold, ${ }^{a}$ Wilcoxon signed rank test

\section{Discussion}

Adenosine stress CMR in patients with MR conditional $\mathrm{PM}$ is complicated by the absence of an inhibited pacing mode in these devices. Choosing an adequate PM setting (deactivation vs. asynchronous pacing) in specific conduction disorders can be challenging due to possible alterations of $\mathrm{HR}$ and heart rhythm under adenosine. Until now no guidelines for clinical routine have been established addressing this issue. We therefore elaborated a protocol for rhythm management intending to minimize the risk of arrhythmia due to competitive PM stimulation on the one hand and possible bradycardia on the other. In this study our protocol proved to be safe and feasible. No complications of adenosine stress CMR related to the presence of a PM or the underlying cardiac conduction disorder occurred.

The present study is in line with our previous findings [13] that in SND with normal resting $\mathrm{HR}$ and normal PQ interval paused PM stimulation (ODO mode) is suitable for adenosine stress perfusion. We found predominance of the sympatho-excitatory effect of adenosine [17] that overrides cardiac inhibition comparable to patients without SND resulting in a significant increase in HR. We selected DOO pacing only in patients with sinusbradycardia $<45 \mathrm{bpm}$ to prevent competitive atrial stimulation which can induce AF [18], also considering the fact that adenosine may promote AF by shortening the atrial action potential and refractory period [19]. Avoiding competitive atrial stimulation by pacing above intrinsic HR is not useful due to the acceleration of HR under adenosine (up to $40 \mathrm{bpm}$ in tis study). Pacing far above baseline HR for a longer time could cause discomfort or even circulatory compromise in PM patients adapted to relative bradycardia [20]. VOO mode was avoided because ventricular tachycardia can be induced by R-on-T PM stimulation [21]. The risk of proarrhythmia with asynchronous ventricular pacing for PM interrogation is considered low [22]. However, routine adenosine stress CMR in CAD requires a long period in MR conditional mode because sequences for localization, cine imaging, first pass perfusion and LGE are necessary. Asynchronous ventricular PM stimulation might be hazardous under these conditions. In patients with higher degree AV-block or bradycardia induced by adenosine in the preceding test the risk of R-on$\mathrm{T}$ stimulation considered low because the patient is paced above intrinsic HR at rest and under adenosine. Prolonged asystole under adenosine stress imaging in occult SND has been reported [23], therefore immediate reactivation of the PM in case of persistent bradycardia must be guaranteed. For further clinical practice we recommend deactivation of the device under careful monitoring in SND without AV-block.

The adenosine test in patients with intermittent AVblock was useful to differentiate patients that can be 
examined with deactivated devices from those with mandatory PM stimulation. One could argue that asynchronous pacing for all patients with AV-block may be chosen. However, with intact AV-conduction under adenosine the risk of proarrhythmia due to competitive ventricular stimulation cannot be excluded. Deterioration of AV-conduction was only observed in patients with both prolonged PQ interval and pathologic Wenckebach point. Larger studies have to show if CMR can be performed without previous testing in patients without first degree AV-block at rest and normal Wenckebach point. We believe that screening for occult AVdysfunction by measuring the PQ-interval and Wenckebach point prior to CMR in SND is useful because SND patients may develop binodal disease [24] that does not become clinically apparent due to the PM therapy. We recommend adenosine test for SND patients with pathologic AV-conduction at the time of CMR, however all individuals in this study had normal AV-conduction. Further studies have to show if adenosine testing is adequate for patients with SND and impaired $\mathrm{AV}$ conduction unmasked prior to CMR.

The investigated pacing protocol showed to be feasible and safe. However, adenosine stress CMR has to be compared to other diagnostic strategies, namely when the high supervisory expense in this setting is considered. We encourage prospective randomized studies to clarify which imaging strategy is the best choice for PM patients in terms of safety and clinical value.

\section{Limitations}

This study is limited by the relatively sample size. Adverse effects may only appear in a larger cohort of patients. Furthermore we did not test a high dose adenosine protocol, which may be more effective in ischemia detection [25].

\section{Conclusion}

Our protocol for rhythm management in patients with MR conditional PM appears to be feasible and safe and may be used for adenosine CMR in clinical routine until larger studies exist.

\section{Abbreviations \\ AV: Atrioventricular; BV: Battery voltage; CAD: Coronary artery disease; CMR: Cardiovascular Magnetic Resonance; ESC: European Society of Cardiology; FLASH: Fast low angle shot; HR: Heart rate; LGE: Late Gadolinium Enhancement; MI: Myocardial infarction; PCT: Pacing capture threshold; PM: Pacemaker; PSIR: Phase-sensitive inversion recovery; SCMR: Society for Cardiovascular Magnetic Resonance; SND: Sinus node dysfunction; SSFP: Steady-state free precession; STIR: Short-tau inversion recovery}

\section{Acknowledgements}

We acknowledge Michael Pilz for editing the graphic content of the manuscript.

\section{Funding}

Not applicable.
Availability of data and materials

Not applicable.

\section{Authors' contributions}

OK conceived of the study, performed the PM interrogations and tests and wrote the manuscript. MG was involved in the conception of the study. GB and RU collected clinical data. MB helped in statistical analysis and literature review and revised the manuscript. KK helped in the conception of the study and in drafting the manuscript. HS was involved in the conception of the study and data analysis. MS was involved in the conception of the study. BH was involved in the conception of the study and revised the manuscript. DG helped in the coordination of the study and revised the manuscript. All authors read and approved the final manuscript.

\section{Authors' information}

OK: Cardiologist, member of the German Cardiac Society (DGK), SCMR and DGK Level III qualification for CMR, DGK-approved for interventional cardiology and special rhythmology. MG: Radiologist, member of the German Röntgen Society, Q2 certificate for CMR. GB: Doctorand, RU: Cardiologist, DGK member. MB:

Physicists, research-, software-, and application specialist for MR. KK: Cardiologist, DGK member, approved for interventional cardiology. HS: Cardiologist, member of the German Cardiac Society (DGK), DGK-approved for interventional cardiology and special rhythmology. MS: Cardiologist, DGK-member, approved for interventional cardiology, SCMR and DGK level III qualification for CMR. BH: Cardiologist, DGK-member, approved for interventional cardiology and special rhythmology. DG: Radiologist, member of the German Röntgen Society, specialist in MR guided interventional therapy.

\section{Competing interests}

The authors declare that they have no competing interests.

\section{Consent for publication}

Not applicable.

\section{Ethics approval and consent to participate}

The study complies with the Declaration of Helsinki and was approved by the ethics committee of the University of Witten/Herdecke. All individuals gave their consent to participate prior to inclusion.

\section{Publisher's Note}

Springer Nature remains neutral with regard to jurisdictional claims in published maps and institutional affiliations.

\section{Author details}

${ }^{1}$ Department of Cardiology, Katholisches Klinikum Essen, University of Witten/Herdecke, Hülsmannstraße 17, 45355 Essen, Germany. ${ }^{2}$ Department of Radiology, Grönemeyer Institut Bochum, University of Witten/Herdecke, Universitätsstraße 142, 44799 Bochum, Germany. ${ }^{3}$ Cardiovascular Center, Josef Hospital, University of Bochum, Gudrunstr. 56, 44791 Bochum, Germany. ${ }^{4}$ Department of Cardiology, Prosper-Hospital Recklinghausen, University of Witten/Herdecke, Mühlenstraße 27, 45659 Recklinghausen, Germany.

Received: 9 February 2017 Accepted: 25 May 2017

Published online: 02 June 2017

\section{References}

1. Nandalur KR, Dwamena BA, Choudhri AF, Nandalur MR, Carlos RC. Diagnostic performance of stress cardiac magnetic resonance imaging in the detection of coronary artery disease: a meta-analysis. J am Coll Cardiol. 2007;50:1343-53.

2. Authors/Task Force $\mathrm{m}$, Windecker S, Kolh P, Alfonso F, Collet JP, Cremer J, et al. 2014 ESC/EACTS guidelines on myocardial revascularization: the Task Force on myocardial revascularization of the European Society of Cardiology (ESC) and the European Association for Cardio-Thoracic Surgery (EACTS) developed with the special contribution of the European Association of Percutaneous Cardiovascular Interventions (EAPCI). Eur Heart J. 2014;35:2541-619.

3. Li M, Zhou T, Yang LF, Peng ZH, Ding J, Sun G. Diagnostic accuracy of myocardial magnetic resonance perfusion to diagnose ischemic stenosis 
with fractional flow reserve as reference: systematic review and metaanalysis. JACC Cardiovasc Imaging. 2014;7:1098-105.

4. Alonso A, Jensen PN, Lopez FL, Chen LY, Psaty BM, Folsom AR, et al. Association of sick sinus syndrome with incident cardiovascular disease and mortality: the atherosclerosis risk in communities study and cardiovascular health study. PLoS One. 2014;9:e109662.

5. Hsueh CW, Lee WL, Chen YT, Ting CT. The incidence of coronary artery disease in patients with symptomatic bradyarrhythmias. Jpn Heart J. 2001;42:417-23.

6. Wollmann CG, Thudt K, Kaiser B, Salomonowitz E, Mayr H, Globits S. Safe performance of magnetic resonance of the heart in patients with magnetic resonance conditional pacemaker systems: the safety issue of the ESTIMATE study. J Cardiovasc Magn Reson. 2014;16:30.

7. Gimbel JR, Bello D, Schmitt M, Merkely B, Schwitter J, Hayes DL, et al. Randomized trial of pacemaker and lead system for safe scanning at 1.5 Tesla. Heart Rhythm. 2013;10:685-91.

8. Schwitter J, Kanal E, Schmitt M, Anselme F, Albert T, Hayes DL, et al. Impact of the Advisa MRI pacing system on the diagnostic quality of cardiac MR images and contraction patterns of cardiac muscle during scans: Advisa MRI randomized clinical multicenter study results. Heart Rhythm. 2013;10:864-72.

9. Bernstein AD, Daubert JC, Fletcher RD, Hayes DL, Luderitz B, Reynolds DW, et al. The revised NASPE/BPEG generic code for antibradycardia, adaptiverate, and multisite pacing. North American Society of Pacing and Electrophysiology/British pacing and electrophysiology group. Pacing Clin Electrophysiol. 2002;25:260-4

10. Biaggioni I, Killian TJ, Mosqueda-Garcia R, Robertson RM, Robertson D. Adenosine increases sympathetic nerve traffic in humans. Circulation. 1991; 83:1668-75.

11. Sweeney MO, Porkolab FL. Ventricular fibrillation induced by double premature ventricular pacing stimuli in a dual-chamber pacemaker with auto capture. Heart Rhythm. 2009;6:429-32.

12. Hesse B, Tagil K, Cuocolo A, Anagnostopoulos C, Bardies M, Bax J, et al. EANM/ESC procedural guidelines for myocardial perfusion imaging in nuclear cardiology. Eur J Nucl med Mol Imaging. 2005;32:855-97.

13. Klein-Wiele O, Garmer M, Urbien R, Busch M, Kara K, Mateiescu S, et al. Feasibility and safety of adenosine cardiovascular magnetic resonance in patients with MR conditional pacemaker systems at 1.5 Tesla. J Cardiovasc Magn Reson. 2015;17:112.

14. Task Force M, Montalescot G, Sechtem U, Achenbach S, Andreotti F, Arden C, et al. 2013 ESC guidelines on the management of stable coronary artery disease: the Task Force on the management of stable coronary artery disease of the European Society of Cardiology. Eur Heart J. 2013;34:29493003.

15. Brignole M, Auricchio A, Baron-Esquivias G, Bordachar P, Boriani G, Breithardt OA, et al. 2013 ESC guidelines on cardiac pacing and cardiac resynchronization therapy: the Task Force on cardiac pacing and resynchronization therapy of the European Society of Cardiology (ESC). Developed in collaboration with the European heart rhythm Association (EHRA). Eur Heart J. 2013;34:2281-329

16. American College of Cardiology Foundation Task Force on Expert Consensus D, Hundley WG, Bluemke DA, Finn JP, Flamm SD, Fogel MA, et al. ACCF/ACR/AHA/NASCI/SCMR 2010 expert consensus document on cardiovascular magnetic resonance: a report of the American College of Cardiology Foundation Task Force on expert consensus documents. J am Coll Cardiol. 2010;55:2614-62.

17. Costa F, Biaggioni I. Adenosine activates afferent fibers in the forearm, producing sympathetic stimulation in humans. J Pharmacol exp Ther. 1993;267:1369-74.

18. Furman S, Cooper JA. Atrial fibrillation during A-V sequential pacing. Pacing Clin Electrophysiol. 1982;5:133-5.

19. Kanei Y, Hanon S, Van-Tosh A, Schweitzer P. Adenosine-induced atrial fibrillation during pharmacologic stress testing: report of eight cases and review of the literature. Int J Cardiol. 2008:129:e15-7.

20. Tops LF, Schalij MJ, Bax JJ. The effects of right ventricular apical pacing on ventricular function and dyssynchrony implications for therapy. J am Coll Cardiol. 2009;54:764-76.

21. Oupadia P, Ramaswamy K. Images in clinical medicine. "R-on- $T^{\prime \prime}$ phenomenon. N Engl J med. 1998;338:1812.

22. Nowak B, Hemmer W, Israel CW, Kramer LI, Neuzner J, Pfeiffer D, et al. Statement of the working Group of the Germany Society on the safety of asynchronous ventricular pacemaker stimulation. Clin res Cardiol. 2006;95:57-60.
23. Pennell DJ, Mahmood S, Ell PJ, Underwood SR. Bradycardia progressing to cardiac arrest during adenosine thallium myocardial perfusion imaging in occult sino-atrial disease. Eur J Nucl med. 1994;21:170-2.

24. Rosenqvist M. Atrial pacing for sick sinus syndrome. Clin Cardiol. 1990;13:43-7.

25. Karamitsos TD, Ntusi NA, Francis JM, Holloway CJ, Myerson SG, Neubauer S. Feasibility and safety of high-dose adenosine perfusion cardiovascular magnetic resonance. J Cardiovasc Magn Reson. 2010;12:66.

\section{Submit your next manuscript to BioMed Central and we will help you at every step:}

- We accept pre-submission inquiries

- Our selector tool helps you to find the most relevant journal

- We provide round the clock customer support

- Convenient online submission

- Thorough peer review

- Inclusion in PubMed and all major indexing services

- Maximum visibility for your research

Submit your manuscript at www.biomedcentral.com/submit
Biomed Central 